\title{
Transition from atomic to molecular adsorption of oxygen on tungsten monomer anion
}

\author{
Davor Stolcic, Young Dok Kim, ${ }^{\text {a) }}$ and Gerd Ganteför \\ Department of Physics, University of Konstanz, D-78457 Konstanz, Germany
}

\begin{abstract}
Using vibrationally resolved ultraviolet photoelectron spectroscopy, we studied oxygen adsorption on $\mathrm{W}$ monomer anions. Three oxygen atoms are atomically bound in a $\mathrm{WO}_{3}{ }^{-}$compound, whereas in $\mathrm{WO}_{4}{ }^{-}$the fourth oxygen atom is attached to the oxygen, forming a di-oxygen species, implying that atom to molecule transition of $\mathrm{O}_{2}$ takes place when the number of oxygen atoms attached to a $\mathrm{W}$ monomer anion exceeds three. Our results indicate that molecular adsorption of oxygen is preferred on electron-deficient metals, showing that the driving force of the atom to molecule transition for the chemisorption of diatomic molecules can be the variations of electronic structures of metal hosts.
\end{abstract}

Metal particles consisting of less than about 15 atoms exhibit completely different electronic, geometric, and chemical properties compared to the larger particles and bulk crystals consisting of the same elements. Consequently, interesting cluster size selectivity can be observed for the chemical reactions. Chemisorption reactivities of $\mathrm{N}_{2}, \mathrm{O}_{2}$, and $\mathrm{H}_{2}$ on various transition metal clusters change by several orders of magnitude as a function of cluster size. ${ }^{1-10} \mathrm{CO}$ adsorption reactivity of metal clusters also shows size effects. ${ }^{11,12}$ Nanoclusters consisting of Au, which is the most inert material as bulk form, becomes extraordinarily active for various catalytic reactions such as low temperature $\mathrm{CO}$ oxidation, and partial oxidation of hydrocarbons. ${ }^{13-15}$ Nanocatalysis is currently one of the most exciting subjects in chemistry and physics.

It has been previously suggested that diatomic molecules are nondissociatively bound on these small nanoclusters. For example, a sharp jump in $\mathrm{N}_{2}$ chemisorption reactivity on $\mathrm{W}_{n}$ ( $n=$ number of atoms) clusters was found at $n=15$, and this result was interpreted in terms of transition from atom (N) to molecule $\left(\mathrm{N}_{2}\right)$ at this cluster size. ${ }^{1-3}$ For $\mathrm{H}_{2}$ on $\mathrm{Ti}_{n}{ }^{-}$clusters, a similar atom to molecule transition was suggested with decreasing cluster size. ${ }^{16}$ To shed light on the mechanisms of the catalytic reactions taking place on nanoclusters, we have recently used high-resolution photoelectron spectroscopy to analyze structures of metal clusters $(\mathrm{W}, \mathrm{Nb}, \mathrm{Ag}$, and $\mathrm{Au}$ ) reacted with $\mathrm{O}_{2}$ or $\mathrm{N}_{2} \cdot{ }^{17-21}$ We provided direct evidence that these diatomic molecules are molecularly bound rather than dissociatively adsorbed. These diatomic species were identified using vibrational fine structures in the UPS spectra, corresponding to the $\mathrm{N}-\mathrm{N}$ or $\mathrm{O}-\mathrm{O}$ stretching frequencies. Chemical reactions on small nanoclusters are suggested to be mediated by the formation of activated diatomic species, rather than the dissociative chemisorption. ${ }^{13,15,18}$ These results are different from those of the metal single crystal surfaces on which these diatomic molecules generally

\footnotetext{
${ }^{a)}$ Author to whom correspondence should be addressed. Electronic mail: young.kim@uni-konstanz.de
}

dissociate at room temperature. ${ }^{22-24}$ The dissociation of these molecules is generally known to be the rate determining step for many catalytic reactions such as CO-oxidation and ammonia synthesis, i.e., reaction mechanisms of nanocatalysis can be much different from those well-established in surface chemistry experiments using metal single crystal surfaces.

What is the origin of the molecular chemisorption of these diatomic molecules on small metal clusters? It has been suggested that changes in electronic structures as a function of clusters size are reflected in the size dependence of the chemical activity. For the dissociative chemisorption of diatomic molecules, charge transfers from metals to the antibonding molecular orbitals of these gas molecules are essential. ${ }^{16,25,26}$ In a very simple electronic model, four and six electrons should be transferred from metal to $\mathrm{O}_{2}$ and $\mathrm{N}_{2}$, respectively, to dissociate these molecules, whereas molecular adsorptions require one or two electrons for $\mathrm{O}_{2}$ and two or four electrons for $\mathrm{N}_{2}$, depending on the adsorption geometry. For the metal clusters consisting of less than 10-20 atoms, the densities of states (DOS) are much lower compared to those of the respective bulk crystals, since continuous metallic band structures are changed to the discrete electronic levels with decreasing cluster size. Lower DOS does not allow a sufficient charge transfer to dissociate diatomic adsorbates, yielding the nondissociative chemisorption. Even though ample evidence has been provided for the chemisorption reactivity of metal clusters being closely related to the electronic structures, one cannot rule out the possibility of the chemisorption being determined by geometric factors. Cluster geometry changes with varying cluster size, yielding new adsorption sites at certain cluster sizes, and this can be responsible for the size dependence in chemisorption reactivity. ${ }^{2}$ Measurements for the chemisorption pattern as a function of cluster size should be interpreted by considering both electronic and geometric factors.

A question can be raised: How far the chemisorption pattern can be influenced by solely electronic (geometric) structures of clusters without considering geometric (electronic) factors? This is actually a long standing question, 
which has been in debate in cluster and surface chemistry, and still remains open. ${ }^{1-10}$ To find direct evidence for the relationship between electronic structure of the metal host and the atom to molecule transition in the chemisorption of diatomic molecules, UPS studies on the $\mathrm{W}$ monomers reacted with oxygen are carried out in the present work. Atomto-molecule transition in $\mathrm{WO}_{n}{ }^{-}$with increasing $n$ (number of oxygen atoms) was found, and this result can be interpreted in terms of electronic structure without taking geometric structures of the adsorption sites into account.

The UPS spectra of $\mathrm{WO}_{3}{ }^{-}$and $\mathrm{WO}_{4}{ }^{-}$created in the pulsed arc cluster ion source (PACIS) were collected using ultraviolet (UV) photon with energies of 6.4 or $4.66 \mathrm{eV}$, and the time-of-flight (ToF) electron spectrometer. ${ }^{27} \mathrm{~A}$ higher photon energy $(6.4 \mathrm{eV})$ was used for $\mathrm{WO}_{3}{ }^{-}$due to the higher electron affinity of this cluster. The cluster temperature in the PACIS source is estimated to be room temperature. The $\mathrm{O}_{2}$ partial pressure is about 0.1 mbar and $\mathrm{O}_{2}$ molecules are dissociated in the electric discharge in the PACIS source, yielding $\mathrm{WO}_{n}{ }^{-}$with $n=$ even numbers as well as odd numbers. Our experimental conditions (high $\mathrm{O}_{2}$ pressure and high concentration of atomic $\mathrm{O}$ in the reagent environments) are sufficient to create the thermodynamic ground state, i.e., the formation of the ground state is most likely not hampered by activation barriers. For example, for larger $\mathrm{W}_{n} \mathrm{O}_{m}{ }^{-}$with $n$ $=2-6$, compounds are formed, in which oxygen atoms are inserted between $\mathrm{W}-\mathrm{W}$ bonds producing $\mathrm{W}-\mathrm{O}-\mathrm{W}$ structures. This kind of $\mathrm{O}$ insertion into the $\mathrm{W}-\mathrm{W}$ bonding should have a high activation energy, however, our cluster temperature and partial pressure of the reagent is high enough to overcome this kinetic barrier. $\mathrm{WO}_{n}{ }^{-}$with $n$ above 5 was not prepared in our experimental conditions.

In Fig. 1, the UPS spectra of $\mathrm{WO}_{3}{ }^{-}$and $\mathrm{WO}_{4}{ }^{-}$are displayed. The $\mathrm{WO}_{3}{ }^{-}$shows a vibrational fine structure of $30 \mathrm{meV}$, which corresponds to the $\mathrm{W}-\mathrm{O}$ stretching frequency, suggesting that oxygen atoms exist in the atomic form. The most probable structure for $\mathrm{WO}_{3}{ }^{-}$is that three oxygen atoms are attached to the $\mathrm{W}$ atoms, forming a triangular structure with a $\mathrm{W}$ atom in the middle of the triangle (Fig. 2). This structure is also confirmed by recent density functional theory (DFT)-calculations. ${ }^{28}$

The UPS spectrum of $\mathrm{WO}_{4}{ }^{-}$gives a periodic structure of $190 \mathrm{meV}$ (Fig. 1), which corresponds to the O-O stretching frequency, suggesting that oxygen is molecularly adsorbed. The structure of $\mathrm{WO}_{4}{ }^{-}$which reconciles the UPS data is demonstrated in Fig. 2, in which two oxygen atoms are separated, whereas two additional oxygen atoms are molecularly attached. Considering that $180 \mathrm{meV}$ is quite close to the corresponding value of an $\mathrm{O}_{2}$ gas molecule (196 $\mathrm{meV}$ ), it is likely that the $\mathrm{O}_{2}-\mathrm{W}$ bonding in $\mathrm{WO}_{4}$ is very weak. Note that the vibrational fine structures of the anion UPS correspond to the vibrational frequencies of the neutral counterparts with similar structures as the anionic clusters. In the anionic state of $\mathrm{WO}_{4}$, an additional electron can occupy the antibonding orbital of $\mathrm{O}_{2}$, further activating the $\mathrm{O}-\mathrm{O}$ bonding with respect to the case of the neutral clusters. ${ }^{18}$

The electron affinity of $\mathrm{WO}_{4}$ is significantly lower than that of $\mathrm{WO}_{3}$. Considering that the dissociate chemisorption requires a much larger charge transfer from metal to adsor-
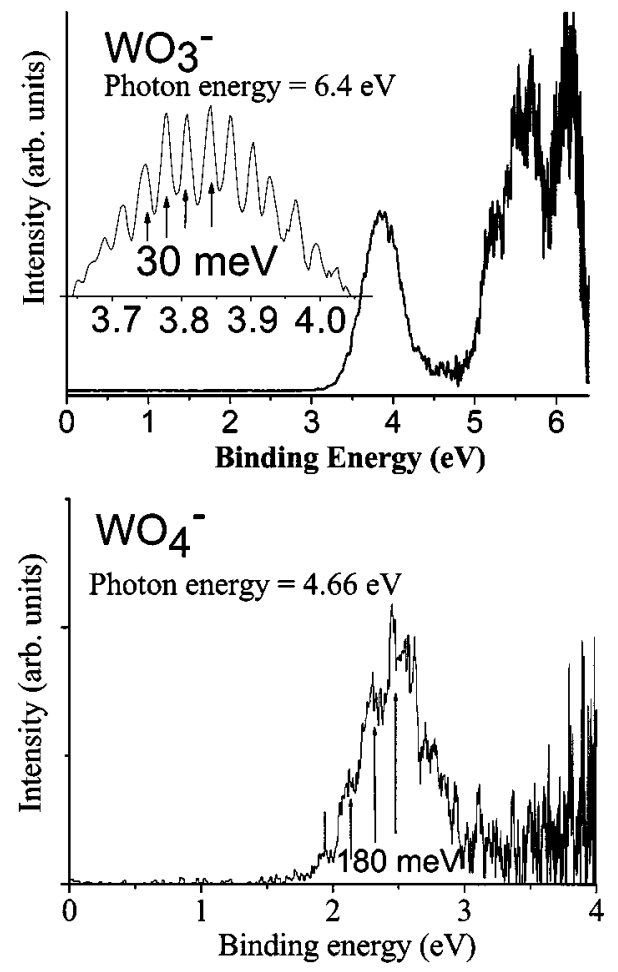

FIG. 1. UPS spectra of $\mathrm{WO}_{3}{ }^{-}$(photon energy $=6.4 \mathrm{eV}$, the magnified spectrum with vibrational fine structures was taken using a photon energy of $4.66 \mathrm{eV})$ and $\mathrm{WO}_{4}{ }^{-}(4.66 \mathrm{eV})$ are displayed.

bate, the lower electron affinity of $\mathrm{WO}_{4}$ than that of $\mathrm{WO}_{3}$ is also indicative of the molecular adsorption of oxygen in $\mathrm{WO}_{4}$.

Between $\mathrm{WO}_{3}{ }^{-}$and $\mathrm{WO}_{4}{ }^{-}$, we have observed the transition from atomic to the molecular adsorption of oxygen. In our experiments using $\mathrm{W}$ monomer anions, the sitespecificity for the dissociative chemisorption does not play a role and the transition from atomic to molecular chemisorption of oxygen between $\mathrm{WO}_{3}{ }^{-}$and $\mathrm{WO}_{4}{ }^{-}$can be solely interpreted in terms of electronic structure. $\mathrm{W}^{-}$has seven electrons in the valence $d$-orbitals. In a very simple electronic model, atomic oxygen withdraws two electrons from the metal host, and therefore three oxygen atoms can be attached to the $\mathrm{W}$ monomer anion, leaving one electron in the $d$-orbital of $\mathrm{W}$. The fourth atomic oxygen cannot be atomically attached to the $\mathrm{W}^{-}$, since there are no sufficient electrons left for the $\mathrm{W} \rightarrow \mathrm{O}$ charge transfer. Thus, in $\mathrm{WO}_{4}{ }^{-}$, the fourth oxygen atom is attached to the oxygen atom of the $\mathrm{WO}_{3}{ }^{-}$cluster, since this does not require additional $\mathrm{W} \rightarrow \mathrm{O}$

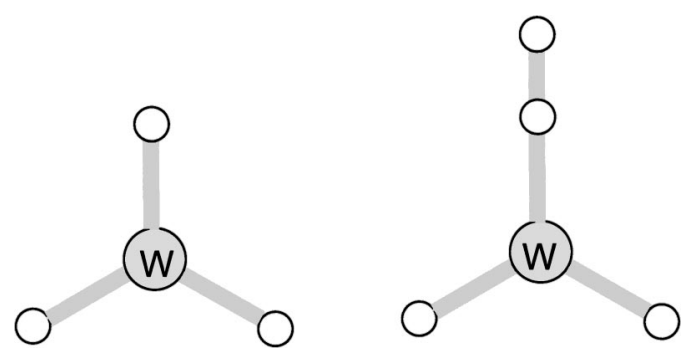

FIG. 2. Structures of $\mathrm{WO}_{3}{ }^{-}$and $\mathrm{WO}_{4}{ }^{-}$suggested based on the UPS results. 
charge transfer with respect to the case of $\mathrm{WO}_{3}{ }^{-}$. Formation of di-oxygen species on electron-deficient metal cluster has been also reported previously. $\mathrm{On} \mathrm{V}, \mathrm{Fe}, \mathrm{Cu}, \mathrm{Cu}_{2}$, and $\mathrm{Al}_{2}$, oxygen is initially atomically bound, however, with increasing number of oxygen atoms formation of di-oxygen species is preferred to the dissociative chemisorption. ${ }^{29-33}$ Formation of di-oxygen-species on O-excess metals has been rationalized in terms of electronic effect, in agreement with the result from the present work. ${ }^{29-33}$

In summary, three oxygen atoms are atomically bound to the $\mathrm{W}$ monomer anion in a $\mathrm{WO}_{3}{ }^{-}$compound, whereas in $\mathrm{WO}_{4}{ }^{-}$the fourth oxygen is attached to another oxygen, forming a di-oxygen species, implying that atom-to-molecule transition of $\mathrm{O}_{2}$ takes place when the number of oxygen atoms attached to a $\mathrm{W}$ monomer anion exceeds three. This result shows that decreased number of valence electrons as a consequence of oxygen adsorption hampers the charge transfer from metal host to oxygen, which is not sufficient to allow the dissociative chemisorption, forming the di-oxygen species in $\mathrm{WO}_{4}{ }^{-}$. This is an example showing that the atomto-molecule transition for the chemisorption of diatomic molecules can be dominated by electronic structures of metal hosts.

DFG (Deutsche Forschungsgemeinschaft) is acknowledged for the financial support.

${ }^{1}$ M. B. Knickelbein, Annu. Rev. Phys. Chem. 50, 79 (1999).

${ }^{2}$ S. A. Mitchell, D. M. Rayner, T. Bartlett, and P. A. Hackett, J. Chem. Phys. 104, 4012 (1996).

${ }^{3}$ L. Holmgren, M. Andersson, and A. Rosěn, J. Chem. Phys. 109, 3232 (1998).

${ }^{4}$ R. L. Whetten, D. M. Cox, D. J. Trevor, and A. Kaldor, Phys. Rev. Lett. 54, 1494 (1985).

${ }^{5}$ J. Conceicao, R. T. Laaksonen, L.-S. Wang, T. Guo, P. Nordlander, and R. E. Smalley, Phys. Rev. B 51, 4668 (1995).
${ }^{6}$ H. Kietzmann, J. Morenzin, P. S. Bechthold, G. Ganteför, and W. Eberhardt, J. Chem. Phys. 109, 2275 (1998).

${ }^{7}$ J. L. Elkind, F. D. Weiss, J. M. Alford, R. T. Laaksonen, and R. E. Smalley, J. Chem. Phys. 88, 5215 (1998).

${ }^{8}$ M. D. Morse, M. E. Geusic, J. R. Heath, and R. E. Smalley, J. Chem. Phys. 83, 2293 (1985).

${ }^{9}$ M. R. Zakin, R. O. Brickman, D. M. Cox, and A. Kaldor, J. Chem. Phys. 88, 3555 (1988).

${ }^{10}$ R. L. Whetten, M. R. Zakin, D. M. Cox, D. J. Trevor, and A. Kaldor, J. Chem. Phys. 85, 1697 (1986).

${ }^{11}$ D. M. Cox, K. C. Reichmann, D. J. Trevor, and A. Kaldor, J. Chem. Phys. 88, 111 (1988).

${ }^{12}$ K. P. Kerns, E. K. Parks, and S. J. Riley, J. Chem. Phys. 112, 3394 (2000).

${ }^{13}$ A. Sanchez, A. Abbet, U. Heiz, W.-D. Schneider, H. Häkkinen, R. N. Barnett, and U. Landman, J. Phys. Chem. A 103, 9573 (1999).

${ }^{14}$ M. Valden, X. Lai, and D. W. Goodman, Science 281, 1647 (1998).

${ }^{15}$ W. T. Wallace and R. L. Whetten, J. Am. Chem. Soc. 124, 7499 (2002).

${ }^{16}$ S. Burkart, N. Blessing, and G. Ganteför, Phys. Rev. B 60, 15639 (1999).

${ }^{17}$ Y. D. Kim, M. Fischer, and G. Ganteför, Chem. Phys. Lett. 377, 170 (2003).

${ }^{18}$ D. Stolcic, M. Fischer, G. Ganteför, Y. D. Kim, Q. Sun, and P. Jena, J. Am. Chem. Soc. 125, 2848 (2003)

${ }^{19}$ Y. D. Kim, D. Stolcic, M. Fischer, and G. Ganteför, Chem. Phys. Lett. 380, 359 (2003)

${ }^{20}$ Y. D. Kim and Gerd Ganteför, Chem. Phys. Lett. (to be published).

${ }^{21}$ Y. D. Kim and Gerd Ganteför, Chem. Phys. Lett. (to be published).

${ }^{22}$ T. Engel and G. Ertl, Adv. Catal. 28, 1 (1979).

${ }^{23}$ The Chemical Physics of Solid Surfaces and Heterogeneous Catalysis, edited by D. A. King and D. P. Woodruff, Vol. 3A (Elservier, B. V. Amsterdam, 1990).

${ }^{24}$ K. Jakobi, Phys. Status Solidi A 177, 37 (2000).

${ }^{25}$ R. Hoffman, Rev. Mod. Phys. 60, 601 (1988).

${ }^{26}$ G. Ganteför, G. Schulze Icking-Konert, H. Handschuhe, and W. Eberhardt, Int. J. Mass Spectrom. Ion Processes 159, 81 (1996).

${ }^{27}$ C.-Y. Cha, G. Ganteför, and W. Eberhardt, Rev. Sci. Instrum. 63, 5661 (1992).

${ }^{28}$ D. Stolcic, Dissertation, Universität Konstanz, 2003.

${ }^{29}$ H. Wu and L.-S. Wang, J. Chem. Phys. 108, 5310 (1998).

${ }^{30}$ L.-S. Wang, H. Wu, and S. R. Desai, Phys. Rev. B 53, 8028 (1996).

${ }^{31}$ H. Wu, S. R. Desai, and L.-S. Wang, J. Am. Chem. Soc. 118, 5296 (1996).

${ }^{32}$ H. Wu, S. R. Desai, and L.-S. Wang, J. Phys. Chem. A 101, 2103 (1997).

${ }^{33}$ S. R. Desai, H. Wu, and L.-S. Wang, J. Chem. Phys. 106, 1309 (1997). 